\title{
Planificación logística en los postgrados de las universidades públicas de la Costa Oriental del Lago
}

\author{
Logistic planning in the postgraduates of the public universities of the East \\ coast of the lake
}

\author{
a- José Hernández \\ josealejandro1807@gmail.com \\ Código ORCID: oooo-ooo1-5359-4170 \\ Universidad del Zulia, Venezuela
}

\section{Resumen}

El estudio buscó describir la planificación del proceso de la gestión logística aplicados en los postgrados de las universidades públicas de la Costa Oriental del Lago. La investigación fue descriptiva, bajo un diseño de campo, no experimental, transeccional. Se consideró como población las tres universidades públicas de la Costa Oriental del Lago. Se empleó la encuesta para la recolección de datos, mediante la aplicación de un cuestionario, conformado por 12 ítems con escala de frecuencia. La validez se realizó a través del juicio de expertos, y para calcular su confiabilidad se empleó el método del Coeficiente Alfa de Cronbach, cuyo valor fue 0,88 . El análisis de los datos se realizó mediante la estadística descriptiva, a través de la media aritmética. Se concluye con una moderada aplicación de la planificación como un proceso a través del cual se establecen los cursos de acción adecuados para alcanzar los objetivos trazados.

\section{Abstract}

The study sought describes the planning of the logistics management process applied in the postgraduate courses of the public universities of the Eastern Coast of the Lake. The research was descriptive, under a field design, not experimental, transectional. The three public universities of the Eastern Lake Coast were considered as population. The survey was used for data collection, through the application of a questionnaire, made up of 12 items with a frequency scale. Validity was carried out through expert judgment, and the Cronbach's alpha coefficient method was used to calculate its reliability, the value of which was 0.88. Data analysis was performed using descriptive statistics, using the arithmetic mean. A moderate application of planning is concluded as a process through which the corresponding courses of action are specified to achieve the established objectives.

\section{Palabras clave:}

Cursos de acción; gestión logística; objetivos; planificación; proceso
Keywords:

Courses of action; logistics management; objectives; planning; process 
INTRODUCCIÓN

Diversos cambios sociales, económicos, políticos, religiosos y culturales están ocurriendo en el ámbito mundial, exigiendo una nueva postura de las organizaciones, las cuales no pueden quedarse observando y dejar que las cosas sucedan sin nada tomar acciones al respecto. De allí que las organizaciones están viviendo una época de grandes cambios, hacia un mundo globalizado, donde se desarrollan momentos de innovación, lo cual implica gran responsabilidad de enfrentar nuevos retos y desafíos del medio.

Estos desafíos actuales han generado exigencias de calidad en distintos ámbitos; por ello es necesario modernizar la gestión en términos generales, promoviendo una mayor eficiencia y el desarrollo de los valores necesarios para el adecuado funcionamiento de las organizaciones.

En tal sentido, una forma de conseguir que las organizaciones sean más productivas, competitivas y rentables, es a través de una adecuada gestión logística, esto implica el mejoramiento continuo de actividades tales como: procesamiento de pedidos, manejo de materiales, distribución de productos terminados, flujo de información entre clientes, procesadores y proveedores así como el direccionamiento organizacional que sirvan para que la organización apalanque el sistema productivo, disminuya costos y cumpla las expectativas de los clientes.

Desde estas perspectivas, la Council of Supply Chain Management Professionals (2013) define la gestión logística como una parte del proceso de la cadena de suministro que se encarga de planificar, ejecutar y controlar de forma eficiente y efectiva el almacenaje y flujo de bienes y servicios así como de toda la información relacionada con estos, entre el punto de origen y de consumo o demanda, a fin de cumplir con las expectativas del consumidor.

En este orden de ideas, se entiende por gestión logística el conjunto de conocimientos, acciones y medios destinados tanto a prever como proveer los recursos necesarios para realizar una actividad principal en tiempo idóneo, forma adecuada, al menor costo, en un marco de productividad y calidad. En este contexto, la gestión logística se ha convertido en una herramienta básica que a través de la planificación, implementación y control busca propiciar el desarrollo estratégico e integral de las empresas ante los cambios del mundo.

Se distingue, como elemento importante del proceso de gestión logística, la planificación que para Sople (2007) tiene la tarea fundamental de definir los objetivos y estrategias del sistema logístico con base en un análisis interno y externo de la situación en que se encuentra la gestión logística en la organización, para luego colocar en la empresa a las personas responsables de las actividades logísticas, de manera que favorezca la coordinación entre ellas.

Atendiendo estas consideraciones, se describe la planificación logística desde el punto de vista gerencial como una estrategia necesaria para manejar de forma integral la cadena de suministros, de tal forma que logre el balance óptimo entre las necesidades del cliente y los recursos disponibles de la empresa y su desempeño debe ser medido a través del servicio al cliente final.

Enmarcada dentro de estos procesos, la educación universitaria enfrenta 
grandes desafíos, uno de ellos es formar profesionales integrales que sean capaces de responder a los cambios acelerados que exige el entorno. Desde esta perspectiva, una de las respuestas para adaptarse ante estos cambios, es la creación de programas de postgrado, lo cual ha venido creciendo vertiginosamente en los últimos años, desarrollando programas en diversas áreas del conocimiento $\mathrm{y}$ atendiendo la formación en niveles de Especialización, Maestría, Doctorado y Postdoctorado.

Al respecto, es importante acotar que en la medida que los postgrados han ido creciendo, se visualizan por una parte estrategias y modalidades institucionales en la búsqueda de dar respuesta a las demandas de conocimiento, y por otra parte se manifiestan insatisfacciones por la calidad del proceso, así como de los resultados esperados por sus participantes.

Enmarcado en este contexto, las universidades públicas, en especial los postgrados de la Costa Oriental del Lago, según el diagnóstico previo realizado por el investigador, se encuentran inmersos en cambios, transformaciones académicas y gerenciales, tanto en las áreas curriculares como también en la función gerencial desde todos los niveles jerárquicos; lo cual ha venido generando exigencias, actividades interpersonales, social y laboral de estas organizaciones.

Como complemento, se observó la carencia de una visión global de los procesos logísticos que han generado ineficiencias en todo el proceso, ya que tanto la información como los materiales no fluyen correctamente. Además de verse afectado el servicio prestado en cuanto a tiempo, calidad, costo y satisfacción de los clientes.

Así las cosas, al comparar estas evidencias, se plantea describir la planificación del proceso de la gestión logística aplicados en los postgrados de las universidades públicas de la Costa Oriental del Lago, para generar recomendaciones para su mejora y en base a ello, llevar a cabo sus operaciones, situando al cliente en el punto central de atención del sistema integral, lo que conducirá a la mejora de los procesos, de la competitividad, e igualmente, generará la reducción de costos llevando los postgrados hacia una sostenida rentabilidad.

\section{Planificación del proceso de la gestión logística}

La planificación es una etapa fundamental en el proceso de gestión, ya que esta se encarga de definir los recursos necesarios para cada función de la organización así como las áreas de atención y de mejoras para lograr los objetivos generales de la empresa, lo cual indudablemente repercutirá en el rendimiento de la organización y en el grado de satisfacción de los clientes. Los aspectos relacionados con la planificación logística incluyen la toma de decisiones sobre las áreas en que se deben invertir los recursos, si se deben expandir o diversificar las operaciones, también conlleva decisiones acerca de cuáles son las estrategias alternativas que proporcionarán mayores beneficios, las cuales determinarán las ventajas competitivas a largo plazo.

En tal sentido, para Sople (2007) la planificación logística comienza por la formulación de la visión y misión logística, la identificación de las oportunidades y amenazas externas de la gestión logística, así como la determinación de las fortalezas y debilidades internas, el establecimiento de 
objetivos a largo plazo y la elección de estrategias específicas a seguir. Esta definición guarda correspondencia con el planteamiento de Serna (2008), quien considera que al nivel de la planificación "se definen una misión, los objetivos y estrategias a mediano plazo, se identifican los proyectos estratégicos tanto a nivel vertical como horizontal y se establecen los planes de acción" (p. 66).

Al respecto, Ballou (2004) considera que la planificación logística es un conjunto de actividades requeridas para mover el producto de manera efectiva y eficiente que aborda cuatro áreas principales: niveles de servicios al cliente, ubicación de las instalaciones, decisiones de inventario y decisiones de transportación, cada una de ellas ejerce un impacto importante sobre el diseño del sistema.

A criterio del investigador, la planificación logística es concebida como un subsistema del perfeccionamiento empresarial a través de un procedimiento que expresa el conjunto de actividades y acciones que se ejecutarán en el proceso logístico, a partir del direccionamiento estratégico de la compañía, de tal forma que se logre la rentabilidad esperada por la compañía.

\section{Niveles de servicio}

Según Álvarez et al. (2006), los niveles de servicio desde la perspectiva de la gestión logística deben encaminarse a la satisfacción de los clientes, que conlleva a importantes consecuencias, tales como: incremento del consumo, recomendación de los servicios de la empresa a clientes potenciales, posibilidad de realizar proyectos comunes con beneficios mutuos $\mathrm{y}$, sobre todo, la seguridad empresarial que todo ello representa.
En este marco de referencia, para Anaya (2014) los estándares de servicio al cliente fijan el nivel de rendimiento y grado de rapidez al cual debe responder el sistema de logística, afectando los costos que apoyan ese nivel de servicio. Fijar requerimientos de servicios muy altos puede forzar los costos de logística hasta llegar a niveles elevados. Por ello, los estándares de servicios deben cooperar con el marketing para lograr determinar las necesidades y requerimientos del cliente para la logística del servicio, además de determinar la respuesta del cliente al servicio y fijar niveles de servicio al cliente. En logística, servicio al cliente, implicará que se obtenga: el grado de certeza, de confiabilidad, de flexibilidad, aspectos cualitativos y mejora continua.

En esta perspectiva, Casanovas y Cuatrecasas (2010) afirman que la política de servicio a clientes es la diferenciación por canales: mayoristas-minoristascliente, o por grandes superficies, rapidez, fiabilidad de entrega y sistemas de información externos. Al respecto, Carranza (2005) afirma que el servicio al cliente es el resultado del sistema logístico y la mercadotecnia. El concepto implica la entrega del producto correcto al consumidor correspondiente en el lugar preciso, en las condiciones adecuadas, en el tiempo indicado y al menor costo total posible, todo lo cual puede ser coordinado, supervisado e implantado tanto por las funciones logísticas como por las de marketing.

En este mismo orden de ideas, para Muñoz (2007) el servicio al cliente es el conjunto de actividades interrelacionadas que ofrece una empresa con el fin de que el cliente obtenga el producto o servicio en el momento y lugar adecuado el cual satisfaga sus necesidades $\mathrm{y} / \mathrm{o}$ 
expectativas, como consecuencia del precio, la imagen y la reputación de la empresa. Un servicio es cualquier actividad o beneficio que una parte pueda ofrecer a otra. Es esencialmente intangible $\mathrm{y}$ no se puede poseer. Su prestación no tiene por qué ligarse necesariamente a un producto físico.

A juicio del investigador, el servicio al cliente juega un papel crítico y de gran importancia, debiendo establecerse cuidadosamente, consecuentemente, si se quiere que los clientes permanezcan leales a sus suministradores habituales. E1 servicio al cliente es sin lugar a dudas, una oportunidad para aprovechar las ventajas de consolidar pedidos de productos a un mismo comprador, reduciendo así el costo económico y administrativo por unidad de producto entregado o por reclamo $\mathrm{o}$ devolución atendida.

Visto así, el servicio al cliente se hace fundamental en el proceso logístico, implicando realizar funciones de servicio que necesitan el marketing y la gestión de la empresa para cumplir con los requerimientos de los clientes, agregando un alto valor añadido al producto, ya que el cliente lo tiene cuando lo necesita y en condiciones determinadas.

\section{Ubicación de instalaciones}

Según Chopra y Meindl (2008), las instalaciones son las ubicaciones físicas reales en la red de la cadena de suministro donde el producto se almacena, ensambla o fabrica. Se clasifican en: sitios de producción y sitios de almacenamiento. Uno de los problemas del diseño de la cadena de suministros comprende las decisiones con respecto al número $\mathrm{y}$ localización instalaciones de producción, la capacidad en cada instalación, la asignación de cada región/ segmento/parte de mercado para una o más locaciones y la selección de proveedores para subensambles, componentes y materiales.

Los autores señalan que el diseño de la cadena de suministro puede consistir en decisiones centralizadas y descentralizadas. En las primeras, las decisiones son coordinadas a través de todas las instalaciones/nodos por su parte en las secundas el gerente de cada instalación/nodo toma sus decisiones de manera independiente.

Para Ballou (2004), las instalaciones son un eslabón fundamental en la estrategia de establecimiento y concentración puesto que la disposición geográfica y su ubicación son el punto de partida para crear un plan de logística. El establecimiento del número, ubicación y tamaño de las instalaciones y la asignación de la demanda del mercado determinarán para ello las rutas por las cuales transitarán los productos al mercado; para este autor la esencia de la estrategia de ubicación de instalaciones radica en el uso más sencillo e inmediato para hacer llegar el/los productos a los consumidores intermedios $\mathrm{y}$ finales, usando medios propicios que van determinados según la ubicación.

De acuerdo con Heizer y Render (2004) las instalaciones pueden considerarse la ubicación física de las oficinas así como también de la fábrica, lo que las distingue es la importancia que se le asigna a cada una y el enfoque que se tenga de dichas instalaciones, las instalaciones orientadas a los procesos buscan minimizar los costos de cargas o viajes y el tiempo relacionados con la distancia, usualmente las instalaciones deben poseer células de trabajos con el fin de agrupar equipos, maquinarias personas 
y así mantener eficiencia y eficacia en las labores evitando la interrupción por la dispersión que suele acontecer en las instalaciones de trabajo.

En consideración con lo expuesto, para el investigador las instalaciones representa el punto de almacenamiento / centro de distribución de una empresa, donde se realizan, se ejecutan y se controlan los planes logísticos y operacionales, que permiten llevar a cabo la actividad dependiendo la razón de ser de la empresa y contempla la interrelación de diversos procesos.

\section{Decisiones de inventario}

Según los postulados de Chopra y Meindl (2008) los inventarios componen toda la materia prima, el trabajo en proceso y producto terminado dentro de la cadena de suministro. El inventario según su clasificación se divide en dos tipos, el primero es el inventario independiente el cual está destinado a satisfacer la demanda de productos terminados del cliente $y$ por último el inventario dependiente, que consiste en materiales o componentes de los productos terminados.

De acuerdo con Sipper y Bulfin (2008), los inventarios son provisiones de materias primas, trabajo en proceso $\mathrm{y}$ productos terminados que dan cabida en numerosos puntos a lo largo del canal de producción y de logística de una empresa, dichos autores los definen cómo: almacenes, patios, y en los estantes de las tiendas de menudeo, entre otros.

Por su parte, Chase et al. (2005) lo definen como la cantidad de existencias de un bien o recurso cualquiera, usado por una organización. De igual manera, el uso de inventarios permite manejar eficientemente los materiales, con ello se logra maximizar la productividad $y$ minimizar las pérdidas, o en el mejor de los casos eliminarlas.

Para Catácora (2006), los inventarios representan las partidas del activo que generan los ingresos para los diferentes tipos de empresas, entre las cuales se encuentran las dedicadas a la transformación de materia prima y las dedicadas a la compra y venta o comercialización de mercancías. El inventario permite almacenar los materiales de acuerdo al tamaño y frecuencia de uso, logrando con ello disponer de existencias en el momento requerido.

Según Narasimhan (2002) un inventario consiste en las existencias de productos físicos que se conservan en un lugar y un momento determinados. Cada artículo distinto del inventario, que se encuentran en algún lugar, se denomina unidad de almacenamiento de existencias (SKU, por las siglas en inglés de Stock Keeling Unit), y cada SKU tiene un número de unidades en existencia. Cada lugar es un centro de almacenamiento.

En opinión de Espinoza (2011) el inventario es una herramienta fundamental en la administración moderna, ya que esta permite a las empresas y organizaciones conocer las cantidades existentes de productos disponibles para la venta, en un lugar y tiempo determinado, así como las condiciones de almacenamiento aplicables en las industrias.

Dentro de este marco de ideas, el investigador consideró que el manejo de inventario es de gran importancia para la gestión logística porque normalmente no es posible, o no es práctico, suministrar producción instantánea o asegurar tiempos de entrega a los clientes. Sirven como amortiguadores entre la oferta y la 
demanda, de manera que se pueda mantener la disponibilidad del producto necesitado para el cliente, a la vez que hay la flexibilidad de producción y logística en la búsqueda de métodos eficientes de fabricación y distribución del producto.

\section{Decisiones de transportación}

Chopra y Meindl (2008) definen el transporte como el encargado de mover el inventario de un punto a otro en la cadena, existen múltiples medios de transporte que junto con sus rutas y las instalaciones, definen la naturaleza de la red de distribución, las decisiones tomadas sobre las directrices logísticas tienen un importante impacto en el ajuste estratégico de una cadena de suministro, ya que estas definen si su naturaleza está orientada a hacerla más eficiente $\mathrm{o}$ a elevar su capacidad de respuesta.

Una mayor cantidad de instalaciones puede mejorar la capacidad de respuesta a los clientes porque se reduce el tiempo de entrega a los mismos, pero implica una mayor cantidad de inventarios distribuidos en las instalaciones y mayores costos de transporte que llega hacia ellas (también llamados inbonud). Una instalación centralizada permite reducir los inventarios pero eleva el tiempo de respuesta a los clientes y los costos de transporte hacia los mismos (también llamados outbound).

En este marco referencial, para Anaya (2014) el transporte ocupa todas las actividades relacionadas directa e indirectamente con la necesidad de ubicar los productos en los puntos de destino correspondientes, de acuerdo con unas condiciones de seguridad, rapidez y costo, elementos que permiten seleccionar el medio de transporte más idóneo para satisfacer tanto las necesidades del cliente como el esfuerzo económico que este representa para la empresa.

Por otra parte, en opinión de Ballou (2004), las decisiones de empleo de transporte incluyen el modo, el tamaño y la ruta, para ello el administrador logístico debe conocer muy bien el tema del transporte, pues incide directamente en factores de competitividad. Es tanta su relevancia, que el grado de desarrollo de una nación puede ser medido en relación con la participación la cual tiene el transporte en la creación de un nivel alto de actividad económica. Con un sistema de transporte poco desarrollado, la amplitud del mercado se limita a las áreas que rodean en forma cercana el punto de producción, esto inhibe la competencia y por tanto el desarrollo competitivo de tales productos y la región.

\section{MÉTODO}

La metodología se centró en el tipo descriptiva, bajo un diseño de campo, no experimental, transeccional. Se consideró como población las tres universidades públicas de la Costa Oriental del Lago: Universidad del Zulia Núcleo COL, Universidad Nacional Experimental Rafael María Baralt y el Instituto Universitario Cabimas, siendo dieciocho (18) sujetos informantes, específicamente doce (12) coordinadores académicos, tres (03) coordinadores administrativos y tres (03) administradores.

Se empleó la encuesta para la recolección de datos, mediante la aplicación de un cuestionario, el mismo quedó conformado por 12 ítems con escala de frecuencia. La validez se realizó a través del juicio de cinco expertos, y para calcular su confiabilidad se empleó el 
método del Coeficiente Alfa de Cronbach, cuyo valor de 0,88 la ubicó como muy alta. El análisis de los datos se realizó mediante la estadística descriptiva, a través del estudio de la media aritmética, utilizando los baremos establecidos para la interpretación del estadístico, que se reseña en la Tabla 1.

Tabla 1. Categoría de análisis para la interpretación del promedio

\begin{tabular}{ccc}
\hline Dimensión & $\begin{array}{c}\text { Rango para la } \\
\text { media }\end{array}$ & Nivel de respuesta para la dimensión y sus indicadores \\
\hline & $4.21-5.00$ & Muy alta aplicación \\
Proceso de & $3.41-4.20$ & Alta aplicación \\
Planificación & $2.61-3.40$ & Moderada aplicación \\
& $1.81-2.60$ & Baja aplicación \\
& $1.00-1.80$ & No hay aplicación \\
\hline
\end{tabular}

Fuente: Elaboración propia.

\section{RESULTADOS}

Como se evidencia en la Tabla 2, se muestra una media para la dimensión de 2,74 indicando moderada aplicación del proceso de la planificación en la gestión logística que se desarrolla en los postgrados de las universidades públicas de la Costa Oriental del Lago. Al detalle, se aprecia que estas instituciones tienen una moderada aplicación de los niveles de servicio $(3,09)$, y de la ubicación de instalaciones $(3,35)$; no obstante con baja aplicación se encuentran las decisiones de inventario $(2,54)$ y de transportación $(1,96)$.

Tabla 2. Dimensión: Planificación del proceso de la gestión logística

\begin{tabular}{lcl}
\hline INDICADOR & $\overline{\boldsymbol{X}}$ & CATEGORIA \\
\hline Niveles de servicio & 3,09 & Moderada aplicación \\
Ubicación de instalaciones & 3,35 & Moderada aplicación \\
Decisiones de inventario & 2.54 & Baja aplicación \\
Decisiones de transportación & 1,96 & Baja aplicación \\
\multicolumn{1}{c}{ DIMENSIÓN } & $\mathbf{2 , 7 4}$ & Moderada aplicación \\
\hline
\end{tabular}

Fuente: Elaboración propia.

Dentro de este contexto, se refleja a criterio del investigador, que en las universidades analizadas el proceso de la planificación de la gestión logística moderadamente se aplican las actividades necesarias para brindar de manera efectiva y eficiente el servicio requerido en los postgrados a fin de lograr sus objetivos, lo cual repercutirá en el rendimiento y en el grado de satisfacción de los clientes.
Al ver los resultados se evidencia, la importancia de que quienes gestionan los postgrados, consideren la planificación de la logística, como una herramienta administrativa que ayuda a incrementar las posibilidades de éxito cuando se quiere alcanzar algo en situaciones de incertidumbre $\mathrm{o}$ de conflictos, que permitan responder la pregunta ¿qué hacer?, en determinadas situaciones como 
la falta de información y de materiales que no fluyen correctamente, todos ejemplos de la planificación de la logística, que fueron diagnosticado al inicio de esta investigación.

Así las cosas, y atendiendo este resultado de moderada aplicación de la dimensión, se evidencia una moderada congruencia con lo expuesto por Sople (2007), para quien a través de la planificación logística se logra la elección de estrategias específicas a seguir. De igual manera moderadamente valida al investigador cuando afirma que es por medio del conjunto de actividades y acciones que se ejecutan a partir de la planificación del proceso de la gestión logística, que se logra la rentabilidad esperada para los postgrados de las universidades públicas de la Costa Oriental del Lago

Ahora bien, al detalle de cada uno de los indicadores utilizados para medir la dimensión a continuación se especifican los resultados obtenidos al respecto, mostrados en la Tabla 3, donde se recoge el resultado de la dimensión y cada uno de sus indicadores. De esta forma, se evidencia moderada aplicación para los indicadores niveles de servicio y ubicación de instalaciones con media de 3,09 y 3,35 respectivamente.

Lo anteriormente descrito se enmarca atendiendo a los valores individuales de cada ítem, al considerar los encuestados con moderada aplicación en cuanto a los niveles de servicio, que estos desde la perspectiva de la gestión logística debe encaminarse a la satisfacción de los clientes, que conlleve a importantes consecuencias, tales como: incremento del consumo, recomendación de los servicios de los postgrados a sus clientes potenciales, tal como lo expresan Álvarez y otros (2006).

Tabla 3. Indicadores de la dimensión planificación del proceso de la gestión logística

\begin{tabular}{|c|c|c|c|c|}
\hline INDICADOR & $\mathbf{N}^{\mathbf{o}}$ & ITEMS & $\overline{\boldsymbol{X}}$ & CATEGORIA \\
\hline \multirow{4}{*}{ 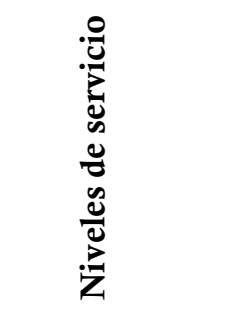 } & 1 & $\begin{array}{l}\text { Se llevan registros de las respuestas del cliente al } \\
\text { servicio recibido }\end{array}$ & 2,61 & Moderada aplicación \\
\hline & 2 & $\begin{array}{l}\text { Brindan información adecuada sobre el producto } \\
\text { vendido. }\end{array}$ & 3,44 & Alta aplicación \\
\hline & 3 & Se crea un buen clima para el servicio al cliente & 3,22 & Moderada aplicación \\
\hline & & INDICADOR & 3,09 & Moderada aplicación \\
\hline \multirow{4}{*}{ 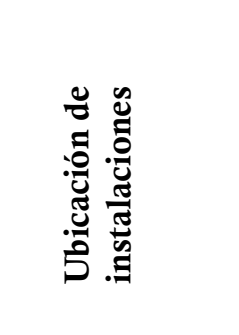 } & 4 & $\begin{array}{l}\text { Cuenta la institución con instalaciones de fácil } \\
\text { acceso }\end{array}$ & 3,33 & Moderada aplicación \\
\hline & 5 & $\begin{array}{l}\text { La capacidad instalada de la institución se } \\
\text { adecuada al número de clientes atendidos }\end{array}$ & 3,28 & Moderada aplicación \\
\hline & 6 & $\begin{array}{l}\text { Las instalaciones de la institución permite } \\
\text { agrupar equipos de trabajo }\end{array}$ & 3,44 & Alta aplicación \\
\hline & & INDICADOR & 3,35 & Moderada aplicación \\
\hline
\end{tabular}




\begin{tabular}{|c|c|c|c|c|}
\hline INDICADOR & $\mathbf{N}^{\mathbf{o}}$ & ITEMS & $\bar{X}$ & CATEGORIA \\
\hline \multirow{4}{*}{ 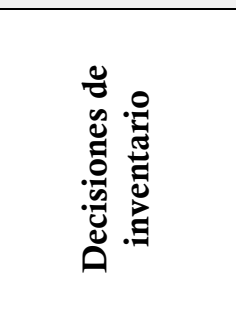 } & 7 & $\begin{array}{l}\text { La institución cuenta con los inventarios para } \\
\text { cumplir con la demanda del mercado }\end{array}$ & 2,67 & Moderada aplicación \\
\hline & 8 & $\begin{array}{l}\text { La institución tiene acceso a toda los recursos } \\
\text { necesarios }\end{array}$ & 2,28 & Baja aplicación \\
\hline & 9 & $\begin{array}{l}\text { Se cuenta con un programa de registro de } \\
\text { inventario }\end{array}$ & 2,67 & Moderada aplicación \\
\hline & & INDICADOR & 2,54 & Baja aplicación \\
\hline \multirow{4}{*}{ 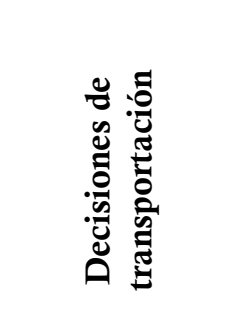 } & 10 & $\begin{array}{l}\text { Cuenta con el transporte adecuado para la } \\
\text { distribución de los recursos }\end{array}$ & 2,00 & Baja aplicación \\
\hline & 11 & $\begin{array}{l}\text { Las operaciones de transporte llevadas a cabo se } \\
\text { realizan sin contratiempos }\end{array}$ & 2,06 & Baja aplicación \\
\hline & 12 & $\begin{array}{l}\text { Se conoce la disponibilidad de vehículo para } \\
\text { realizar los movimientos de recursos }\end{array}$ & 1,83 & Baja aplicación \\
\hline & & INDICADOR & 1,96 & Baja aplicación \\
\hline
\end{tabular}

Fuente: Elaboración propia.

En relación a la ubicación de instalaciones, al situarse en moderada aplicación se hace necesario contar con oficinas acordes a los procesos y que permitan agrupar equipos para mantener con eficiencia y eficacia las labores, evitando la interrupción por la dispersión que suele acontecer en las instalaciones de trabajo, según la teoría reseñada por Heizer y Render (2004).

Respecto a los indicadores decisiones de inventario y decisiones de transportación se ubicaron en la categoría de baja aplicación, con medias de 2.54 y 1,96 respectivamente, implicando con ello que en los postgrados de las instituciones analizadas en su proceso de planificación logística el uso de los inventarios no permite manejar eficientemente los materiales, por lo que no se logra maximizar su productividad, no validando así lo expuesto por Chase et al. (2005), como tampoco se cuenta con el transporte más idóneo para satisfacer tanto las necesidades del cliente como el esfuerzo económico que este representa para los postgrados de ubicar los requerimientos en los puntos de destino correspondientes, de acuerdo con unas condiciones de seguridad, rapidez y costo, de manera, que so existe congruencia con lo expuesto por Anaya (2014).

En resumen, los resultados mostrados evidencian la necesidad de implementar una planificación de la gestión logística que permita realizar un adecuado proceso administrativo en los postgrados para lograr el ajuste perfecto entre la estrategia corporativa y la estrategia de la cadena de suministro, partiendo de la identificación de las necesidades de los clientes a fin de garantizar lo demandado en tiempo, calidad y costos competitivos.

\section{CONCLUSIONES}

En lo concerniente al objetivo, donde se describe la planificación del proceso de la gestión logística aplicados en los postgrados de las universidades públicas de la Costa Oriental del Lago, se afina una moderada aplicación de la planificación como un proceso a través del cual se establecen los cursos de acción adecuados para alcanzar los objetivos trazados en los postgrados de las instituciones analizadas. 
Al detalle se observó, una moderada aplicación de los niveles de servicio y de la ubicación de instalaciones. No obstante con baja aplicación se encuentran las decisiones de inventario y de transportación, actividades necesarias que deben ser consideradas en este proceso de la gestión logística para brindar de manera efectiva y eficiente el servicio requerido en los postgrados a fin de lograr sus objetivos, lo cual repercutirá en el rendimiento y en el grado de satisfacción de los clientes.

\section{REFERENCIAS}

Álvarez, J., Álvarez, I. y Bullón, J. (2006). Introducción a la calidad. Aproximación a los sistemas de gestión y herramientas de calidad. Primera edición. Editorial Ideas propias. España

Anaya, J. (2014). E1 diagnóstico logístico: Una metodología para promover mejoras competitivas. Libros profesionales de empresa. Libros profesionales de empresa. Editorial ESIC. Madrid. España

Ballou, R. (2004). Logística. Administración de la cadena de suministro. Quinta Edición. Editorial Pearson Educación. México

Carranza, O. (2005). Logística: Mejores prácticas en Latinoamérica. Publicado por International Thomson. México

Casanovas, A. y Cuatrecasas, L1. (2010). Logística empresarial. Sexta edición. Ediciones Gestión 2000 S.A. Barcelona. España

Catácora, F. (2006). Contabilidad. La base para las decisiones gerenciales.
Editorial: $\quad$ McGraw Hill Interamericana de Venezuela

Chase, R., Aquilano, N. y Jacobs F. (2005). Administración de la Producción y Operación para una ventaja Competitiva. Décima Edición. Editorial McGraw Hill. México D.F, México

Chopra, S., Meindl, P. (2008). Administración de la Cadena de Suministro, Estrategia, Planeación y Operación. Tercera Edición. Pearson Educación. México D.F, México

Council of supply chain management professionals (2013). Logistics management. Disponible en: http://cscmp.org/about-us/supplychain-management-definitions Consultado: 29/01/2020

Espinoza, O. (2011). La Administración Eficiente de los Inventarios. Primera edición Editorial la Ensenada Madrid

Heizer, J. y Render, B. (2004). Principios de Administración de Operaciones. 5ta Edición. Prentice Hall Hispanoamericana. Madrid

Long, D. (2006) Logística Internacional. Administración de la cadena de abastecimiento. Editorial Noriega Limusa. México D.F, México

Muñoz, A. (2007) La gestión de calidad total en la administración pública. Ediciones Díaz de Santos. Madrid

Narasimhan, S. (2002).Planeación de la Producción y Control de Inventario. Editorial: Prentice-Hall. México

Sipper, D. y Bulfin, R. (2008). Planeación y control de la producción. Editorial McGraw Hill. México

Sople, V. (2007). Logistics management: the supply chain imperative. 2nd ed., Ed. Pearson education, pp. 204-211. India 